\title{
Registro de información en pacientes con trastornos por uso de alcohol en Mar del Plata, Argentina
}

\author{
Karina Conde ${ }^{\star}$, Milena Laura Remaggi ${ }^{* *}$, Raquel Inés Peltzer ${ }^{* * *}$, Aldana Lichtenberguer ${ }^{* * *}$, \\ Mariana Cremonte ${ }^{* * * *}$
}

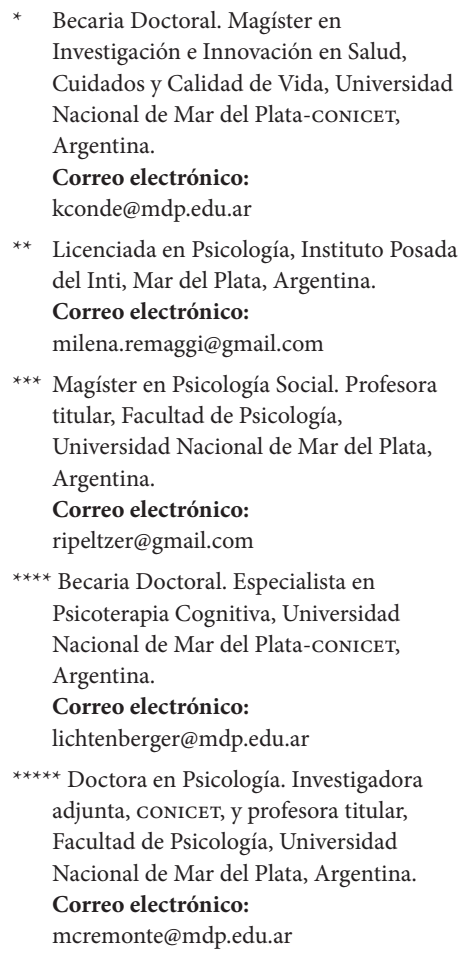

Recibido: 17 de febrero del 2016 Aprobado: 24 de mayo del 2016

Cómo citar este artículo: Conde, $\mathrm{K}$, Remaggi, M. L., Peltzer, R. I., Lichtenberger, A. y Cremonte, M. (2016). Registro de información en pacientes con trastornos por uso de alcohol en Mar del Plata, Argentina. Pensando Psicología, 12(20), 43-52. doi: http://dx.doi.org/10.16925/pe.v12i20.1562

\section{Resumen}

Introducción: los datos que se registran sobre el consumo de alcohol son importantes para el diagnóstico y pronóstico de trastornos por uso de alcohol. Objetivo: este estudio descriptivo tuvo como objetivo caracterizar las referencias sobre consumo de alcohol y otras variables relacionadas en los registros hospitalarios de pacientes atendidos en una unidad especializada en alcoholismo de un hospital público de la ciudad de Mar del Plata, Argentina. Metodología: se examinaron los registros de 60 admisiones entre febrero y noviembre del 2011, y se categorizaron extrayendo datos referidos al consumo de alcohol, fuente de la derivación, diagnóstico, tratamiento, internaciones y otras enfermedades, así como aspectos del entorno familiar y socioeconómico. Posteriormente, se realizaron análisis descriptivos teniendo en cuenta el porcentaje de registros en los que se mencionaban estos aspectos. Resultados: si bien el registro de datos referentes al consumo de alcohol fue alto, por lo general fue incompleto. La información registrada denotó que las condiciones biopsicosociales en las que se encuentran estos pacientes son poco favorables para un tratamiento exitoso. Conclusiones: se discute la necesidad de sistematizar la información, por ejemplo, a través de historias clínicas unificadas, junto con formas de prevenir la progresión de trastornos por uso de alcohol.

Palabras clave: Argentina, atención hospitalaria, registros, trastorno por uso de alcohol. 


\title{
Recording Data of Patients with Alcohol Use Disorders in Mar del Plata, Argentina
}

\begin{abstract}
Introduction: Data recorded on alcohol consumption are important for the diagnosis and prognosis of alcohol use disorders. Objective: This descriptive study aimed to characterize references to alcohol consumption and other related variables in hospital records of patients treated in a specialized alcoholism unit at a public hospital in Mar del Plata, Argentina. Methodology: Records of 60 admissions between February and November 2011 were examined and categorized by extracting data related to alcohol consumption, source of referral, diagnosis, treatment, hospitalization, and other diseases, as well as aspects of family and socioeconomic environment. Subsequently, a descriptive analysis was conducted considering the percentage of records mentioning these aspects. Results: While the recording of data regarding alcohol consumption was high, it was generally incomplete. Recorded data made evident that the bio-psycho-social conditions of these patients are unfavorable for successful treatment. Conclusions: The need to systematize information (for example, through unified medical records) and ways to prevent progression of alcohol use disorders are discussed.
\end{abstract}

Keywords: Argentina, hospital care, records, alcohol use disorder.

\section{Registro de informação em pacientes com transtornos por uso de álcool em Mar del Plata, Argentina}

\section{Resumo}

Introdução: os dados registrados do consumo de álcool são importantes para o diagnóstico e prognóstico de transtornos por uso de álcool. Objetivo: este estudo descritivo teve como escopo caracterizar as referências sobre consumo de álcool e outras variáveis relacionadas nos registros hospitalares de pacientes atendidos em uma unidade especializada em alcoolismo de um hospital público da cidade de Mar del Plata, Argentina. Metodologia: foram avaliados os registros de 60 admissões entre fevereiro e novembro de 2011, e foram categorizados tirando dados referidos ao consumo de álcool, fonte de derivação, diagnóstico, tratamento, internações e outras doenças, bem como elementos do ambiente familiar e socioeconómico. Posteriormente, analises descritivas foram aplicadas considerando a percentagem de registros onde eram indicados tais aspectos. Resultados: embora o registro de dados referentes ao consumo de álcool fosse elevado, em geral foi incompleto. A informação cadastrada evidenciou que as condições biopsicossociais nas quais se encontram esses pacientes são pouco favoráveis para ter sucesso no tratamento. Debate-se a necessidade de sistematizar a informação, por exemplo, através de históricos clínicos unificados, junto com formas de prevenir a progressão de transtornos por uso de álcool.

Palavras chave: Argentina, atenção hospitalar, registros, transtorno por uso de álcool. 


\section{Introducción}

A nivel mundial, el consumo patológico de alcohol es un factor de riesgo para más de 60 tipos de enfermedades. Entre estas se encuentran cirrosis, epilepsia, intoxicaciones, lesiones por violencia y producto de siniestros viales, y varios tipos de cáncer (World Health Organization, 2014). En Argentina, es el primer factor de riesgo de enfermedad y discapacidad (Monteiro, 2007). Por estas razones, las admisiones hospitalarias por patologías relacionadas con el consumo de alcohol son frecuentes y se utilizan muchos recursos de los hospitales (Cherpitel et al., 2006), por lo cual resultan costosos los tratamientos (Marik y Mohedin, 1996; Zachry et al., 2002). En los servicios sanitarios el consumo problemático de alcohol es identificado tardíamente (Monras et al., 2003), cuando las complicaciones psíquicas y sociofamiliares son tan graves que dificultan un tratamiento adecuado. Esto empeora el pronóstico tanto del trastorno por uso de alcohol (Monras et al., 2004), como de las demás enfermedades orgánicas asociadas. Es así que cuando la derivación se hace desde los servicios de salud, generalmente se trata de pacientes con serios daños físicos y psicosociales (Atkinson et al., 2003). La detección temprana en la atención sanitaria también es importante, ya que ocultar trastornos por uso de alcohol puede tener efectos en los tratamientos médicos, por ejemplo, interfiriendo con ciertas medicaciones (Smothers et al., 2004).

Cuando una persona con un trastorno por uso de alcohol finalmente busca tratamiento, no sería por esta razón, ni por tener consecuencias más severas derivadas del consumo de alcohol, sino por la comorbilidad con otros trastornos. Esto podría deberse tanto a que no relacionan las consecuencias adversas con su ingesta de alcohol (Proudfoot y Teesson, 2002), como al estigma social relacionado con la búsqueda de tratamiento en algunos grupos (Esper et al., 2013). Hay estudios que sugieren que si los pacientes no han sido hospitalizados de manera clara por uso patológico de alcohol, la información en los registros médicos sobre un diagnóstico de trastorno por uso de alcohol es escasa (Esper et al., 2013; Nielsen y Gluud, 1992). Adicionalmente, se ha reportado que en pacientes internados en hospitales, alrededor de la mitad de los registros de ingreso tendrían alguna referencia al consumo de alcohol, por lo general incompleta (Pechansky et al., 2005). De igual forma, se ha reportado que las historias clínicas de personas con un diagnóstico positivo de trastorno por uso de alcohol en pocos casos exhibirían datos sobre la ingesta de alcohol y/o variables relacionadas con el pronóstico del trastorno (Smothers et al., 2004).

En resumen, el uso perjudicial de alcohol está frecuentemente relacionado con los ingresos hospitalarios y las consultas médicas, aunque la información sobre este uso no suele ser mencionada en los registros de los pacientes. En nuestro contexto, no se han encontrado estudios que traten el tema, aun cuando se ha visto que casi uno de cada diez pacientes atendidos en servicios de urgencias de hospitales padecen dependencia del alcohol según el Manual Diagnóstico Estadístico de Trastornos Mentales (DSM-5); y una prevalencia total de trastornos por uso de alcohol de 17\% (Cremonte et al., 2010). El objetivo de este trabajo es describir cuánta y qué información es documentada, en los registros hospitalarios de pacientes atendidos en una unidad de hepatopatía y alcoholismo de la ciudad de Mar del Plata, sobre el uso patológico de alcohol y variables relacionadas con él. Se espera que los resultados revelen la necesidad de disponer de dicha información no solo a nivel regional, sino también en otros países con características similares de funcionamiento de los servicios de salud.

\section{Método}

\section{Tipo de estudio}

Se trató de un estudio descriptivo en el que, retrospectivamente, se revisaron historias clínicas y otros registros informales de pacientes (referiremos ambas cosas en el resto del artículo como registros) admitidos en la Unidad de Diagnóstico y Tratamiento de Hepatología y Alcoholismo (UDTHA) de un establecimiento sanitario provincial ubicado en la ciudad de Mar del Plata, el cual asiste casos de alta complejidad de 16 partidos de la provincia de Buenos Aires, Argentina. Esta provincia se divide territorial y administrativamente en 135 partidos, cada uno de los cuales comprende una o más localidades. La UDTHA fue creada en 1985. Esta unidad es un equipo interdisciplinario de médicos internistas, psicólogos, enfermeros y trabajadores sociales que cumplen voluntariamente funciones de asistencia ambulatoria a personas con un trastorno por uso de alcohol, y de investigación y docencia en la temática del alcoholismo (Arzeno et al., 1994). 


\section{Muestra}

Los resultados presentados corresponden a 60 registros de pacientes admitidos por primera vez, seleccionados sistemáticamente entre febrero y noviembre del 2011 según la lista de turnos de la UDTHA. Los datos se recolectaron en forma anónima y se obtuvieron datos sociodemográficos como la edad, el sexo, el estado civil, el nivel educacional, la cobertura en salud, la ocupación y la estabilidad laboral. Del total, seis registros fueron de mujeres (10\%, IC 95\% [2-18]), y 54 de hombres (90\% IC 95\% [82-98]). La edad media global fue de $47,32( \pm 10,99)$ años; $51,5( \pm 8,69)$ años para mujeres y $46,85( \pm 11,19)$ años para hombres. La tabla 1 resume los datos descriptivos.

\section{Tabla 1}

Características sociodemográficas de pacientes atendidos en una unidad de alcoholismo, Mar del Plata, Argentina, 2011

\begin{tabular}{lc}
\hline \multicolumn{1}{c}{ Variable } & $\%($ IC 95\%) \\
\hline Estado civil & $27(12-42)$ \\
Soltero & $29(14-44)$ \\
Casado/Unido de Hecho & $37(21-53)$ \\
Divorciado/Separado & $7(2-20)$ \\
Viudo & \\
\hline Nivel educativo & $10(1-32)$ \\
Primario Incompleto & $30(12-54)$ \\
Primario Completo & $45(23-69)$ \\
Secundario Incompleto & $10(1-32)$ \\
Secundario Completo & $5(13-25)$ \\
Universitario Completo & \\
\hline Ocupación & $36(20-52)$ \\
Desocupado & $28(13-44)$ \\
Empleado & $20(7-35)$ \\
Cuentapropista & $8(2-21)$ \\
Subempleado & $3(0-14)$ \\
Jubilación/Pensión & $5(1-17)$ \\
Ayuda Familiar & \\
\hline Estabilidad laboral & $52(31-73)$ \\
Sí & $22(9-42)$ \\
No & \\
\hline Cobertura & \\
Pública & \\
Obra social & \\
\hline & \\
\hline & \\
\hline &
\end{tabular}

Nota . IC = Intervalo de confianza de porcentajes. Elaboración propia.
Los restantes datos cualitativos fueron categorizados en cuatro grandes grupos, compuestos por las siguientes variables:

1. Consumo de alcohol: (a) Edad de inicio de consumo de alcohol. Contempló el registro de la edad en que el paciente comenzó a consumir alcohol. Se trató de una variable continua en años. (b) Frecuencia, cantidad y bebida típica. La frecuencia estuvo compuesta por los valores todos los días, casi todos los días, tres o cuatro veces a la semana, una o dos veces por semana, dos o tres veces al mes y cerca de una vez al mes. La cantidad se estimó en litros, al no ser posible establecer el número de unidades estándar. A fin de obtener una orientación en este sentido, se tuvo en cuenta el tipo bebida alcohólica más consumida. (c) Enfermedades orgánicas relacionadas directamente con el consumo de alcohol. La presencia de enfermedades como cirrosis, várices esofágicas, encefalopatía, síndrome ascítico edematoso (SAE), peritonitis bacteriana espontánea (PBE), y el grado de disfunción hepática según su clasificación en CHILD A, B o C (Child, 1964), fue estimada e incluida en una variable dicotómica que indicó si el paciente tenía al menos una de las anteriores.

2. Derivación, diagnóstico y tratamiento: (a) Derivación. Se consideraron cuatro fuentes de derivación: médica, judicial, voluntad propia y otros (familiares y Alcohólicos Anónimos). (b) Motivo de consulta. Con base en lo registrado por los profesionales, se establecieron los siguientes cuatro motivos de consulta: uso de alcohol (el consumo de alcohol era la preocupación mayor), problemas psicosociales y uso de alcohol, síntomas de trastornos por uso de alcohol, orden judicial y control/no especificado. (c) Diagnóstico presuntivo. Se observaron cuatro tipos de diagnósticos en los registros: trastorno por consumo de alcohol, trastorno por uso de sustancias (incluido el alcohol), hepatopatía y problemas psicosociales. (d) Otros tratamientos y recursos. La concurrencia a grupos de Alcohólicos Anónimos (AA) o al Centro Provincial de Atención en Adicciones (CPA), el tratamiento por médicos clínicos y las sesiones psicoterapéuticas individuales o grupales fueron considerados tanto si eran concurrentes como pasados. Cuando se aplicó al caso, se exploraron los motivos de abandono y se agruparon en recaídas y otros (trabajo y poca adherencia). 
(e) Indicaciones. Se consideró si el profesional indicaba una dieta, asistencia a grupos, concurrencia al servicio de salud mental $u$ otro, y si era medicado.

3. Internaciones y otras enfermedades: (a) Internaciones previas. Las admisiones previas en las unidades de internación fueron detectadas y sus motivos fueron incluidos en cinco categorías: intoxicación alcohólica/alcoholismo, síndrome de abstinencia, infecciones/hemorragia digestiva/pancreatitis, lesiones y otros. Por su parte, los síntomas de ingreso se clasificaron en tres grupos: temblores, sudoración, excitación, delirio y/o insomnio, diarrea, hemorragia digestiva o dolor abdominal, y varios. (b) Otras enfermedades. Se tuvo en cuenta la presencia/ausencia de otras enfermedades.

4. Entorno del paciente: (a) Convivencia. Se crearon cuatro categorías: solo, familia propia (cónyuge y/o hijos propios o familia ensamblada), familia de origen (madre, padre, hermanos), institucionalizado/otro (hogar, refugio, casa de amigos, etc.). (b) Continencia familiar. Se trató de una variable dicotómica que fue valorada con base en la observación que los profesionales de unidad hicieron del apoyo familiar en el inicio y sostenimiento de un tratamiento. (c) Antecedentes familiares de consumo. Conformada por tres categorías: un familiar directo (padre o madre o hermano), varios familiares (padre y/o hermanos y/o madre y/o tíos y/o abuelos), y ninguno/desconoce.

También se tuvieron en cuenta las recaídas durante los tratamientos realizados, la experiencia en distintos grupos de autoayuda, y el consumo pasado y actual de otras sustancias psicoactivas (i.e. cannabis, cocaína, heroína, tabaco, alucinógenos, sedantes y otras).

\section{Procedimiento}

Los registros fueron facilitados por el hospital en el marco de un convenio de cooperación con la Facultad de Psicología de la Universidad Nacional de Mar del Plata. Accedieron a los registros dos investigadoras que hicieron una revisión simultánea y establecieron criterios de codificación desarrollados a priori. Luego se discutió su adecuación en el curso de la codificación en un proceso iterativo, y se llegó a un acuerdo sobre las categorías finales.

\section{Análisis de datos}

Se efectuaron análisis descriptivos de frecuencias. Las estimaciones se realizaron con los paquetes estadísticos spss versión 12.0 y Epidat 3.1 en sus versiones para Windows.

\section{Consideraciones éticas}

La confidencialidad de los datos fue debidamente respetada. Ninguna historia clínica se transportó a otro establecimiento y solo accedieron a ellas las investigadoras, quienes no mantenían ninguna relación con los pacientes. No se realizó ningún tipo de intervención en los pacientes.

\section{Resultados}

De los registros observados, el 70\% (IC 95\% [58- 82]) tenía algún tipo de información referida al grupo de consumo de alcohol; el 100\% (IC 95\% [94-100]) al de derivación, diagnóstico y tratamiento; el 78\% (IC 95\% [67-90]) al de internaciones y otras enfermedades, y el 72\% (IC 95\% [59-84]) al de entorno familiar. La media de edad de inicio en el consumo de alcohol fue apenas superior al límite legal de consumo. Sin embargo, la edad de inicio de consumo más frecuente fue 20 años (18\%, IC 95\% [6,76-34,53]). La frecuencia de ingesta fue mayormente diaria, y en uno de cada 10 registros se observaron enfermedades relacionadas directamente con el consumo de alcohol. De un $67 \%$ (IC 95\% [54-79]) que lo indicaba, cerca de un tercio presentaba recaídas durante el tratamiento (35\%, IC 95\% [22- 47]). La experiencia en grupos de autoayuda fue del 78\% (IC 95\% [63-92]) en los casos en que se informó este dato. Un gran porcentaje de las derivaciones las realizó el médico y se diagnosticó inicialmente un trastorno por consumo de alcohol en la mayoría de los casos (ver tabla 2).

En cuanto al consumo de otras sustancias, fue registrado en el 64\% de los casos (IC 95\% [49-80], siendo la prevalencia actual de 45\% (IC 95\% [26-64]). La sustancia más utilizada fue el tabaco, seguido por la cocaína y el cannabis.

\section{Discusión}

En este trabajo, se examinó la información contenida en los registros hospitalarios de los pacientes atendidos en una unidad de alcoholismo, en busca de referencias al consumo patológico de alcohol y variables 
Tabla 2

Información disponible en los registros de pacientes atendidos en la unidad de alcoholismo, Mar del Plata, Argentina, 2011

\begin{tabular}{|c|c|c|c|c|c|}
\hline \multirow{2}{*}{ Variables } & \multicolumn{3}{|c|}{ Con información } & \multicolumn{2}{|c|}{ Sin información } \\
\hline & $\%^{*}$ & M (Ds) & IC $95 \%$ & $\%$ & IC $95 \%$ \\
\hline \multicolumn{6}{|l|}{ Consumo de alcohol } \\
\hline Edad de inicio & --- & $18,94(8,47)$ & $15,99-21,9$ & 43 & $30-57$ \\
\hline Frecuencia & & & & 65 & $52-78$ \\
\hline Todos los días & 86 & --- & $64-97$ & --- & --- \\
\hline Una o dos veces por semana & 14 & --- & $3-36$ & --- & --- \\
\hline Cantidad en litros & --- & $4,26(2,68)$ & $2,97-5,55$ & 68 & $56-81$ \\
\hline Bebida típica & & & & 83 & $73-94$ \\
\hline Vino & 40 & --- & --- & --- & --- \\
\hline Cerveza & 60 & --- & --- & --- & --- \\
\hline $\begin{array}{l}\text { Enfermedades relacionadas directamente con } \\
\text { el consumo de alcohol }\end{array}$ & 12 & --- & $3-21$ & 88 & $79-97$ \\
\hline \multicolumn{6}{|l|}{ Derivación, diagnóstico y tratamiento } \\
\hline Derivación & & & & 45 & $32-58$ \\
\hline Juez & 18 & --- & --- & --- & --- \\
\hline Propia voluntad & 30 & --- & --- & --- & --- \\
\hline Médico & 36 & --- & --- & --- & --- \\
\hline Familia/AA/Otro & 15 & --- & --- & --- & --- \\
\hline Motivo de consulta & & & & 30 & $18-42$ \\
\hline Uso de alcohol & 19 & --- & --- & --- & --- \\
\hline Problemas psicosociales y uso de alcohol & 12 & --- & --- & --- & --- \\
\hline Síntomas de trastornos por uso de alcohol & 29 & --- & --- & --- & --- \\
\hline Control/No especificado & 33 & --- & --- & --- & --- \\
\hline Orden judicial & 7 & --- & --- & --- & --- \\
\hline Diagnóstico presuntivo & & & & 28 & $16-41$ \\
\hline Trastornos por uso de alcohol & 74 & --- & $60-89$ & --- & --- \\
\hline Hepatopatía & 9 & --- & $3-22$ & --- & --- \\
\hline Trastorno por uso de sustancias & 12 & --- & $4-25$ & --- & --- \\
\hline Problemas psicosociales & 5 & --- & $0-16$ & --- & --- \\
\hline \multicolumn{6}{|l|}{ Otros tratamientos y recursos } \\
\hline Actuales & & & & 60 & $47-73$ \\
\hline Grupo AA & 52 & --- & --- & --- & --- \\
\hline Médico clínico & 25 & --- & --- & --- & --- \\
\hline Psicoterapia individual & 25 & --- & --- & --- & --- \\
\hline Psicoterapia grupal & 19 & --- & --- & --- & --- \\
\hline Previos & & & & 55 & $42-68$ \\
\hline Grupo AA & 58 & --- & --- & --- & --- \\
\hline Médico clínico & 11 & --- & --- & --- & --- \\
\hline Psicoterapia individual & 27 & --- & --- & --- & --- \\
\hline Psicoterapia grupal & 17 & --- & --- & --- & --- \\
\hline Grupo CPA & 16 & --- & --- & --- & --- \\
\hline
\end{tabular}


(viene)

\begin{tabular}{|c|c|c|c|c|c|}
\hline \multirow{2}{*}{ Variables } & \multicolumn{3}{|c|}{ Con información } & \multicolumn{2}{|c|}{ Sin información } \\
\hline & $\%^{*}$ & M (Ds) & IC $95 \%$ & $\%$ & IC $95 \%$ \\
\hline Abandono tratamientos previos & 56 & --- & --- & 73 & $61-85$ \\
\hline Motivo de abandono & & & & 78 & $67-90$ \\
\hline Recaída & 23 & --- & --- & --- & --- \\
\hline Otro (trabajo, poca adherencia) & 23 & --- & --- & --- & --- \\
\hline Indicaciones & & & & 67 & $54-79$ \\
\hline Dieta & 41 & --- & --- & --- & --- \\
\hline Asistencia a Grupos de AA & 41 & --- & --- & --- & --- \\
\hline Tratamiento psiquiátrico & 12 & --- & --- & --- & --- \\
\hline Otros & 6 & --- & --- & --- & --- \\
\hline Medicación & 88 & --- & --- & 45 & $32-58$ \\
\hline \multicolumn{6}{|l|}{ Internaciones y otras enfermedades } \\
\hline Internaciones previas & 82 & --- & $68-95$ & 37 & $24-50$ \\
\hline Motivos & & & & 47 & $33-60$ \\
\hline Intoxicación alcohólica/Alcoholismo & 22 & --- & $6-38$ & --- & --- \\
\hline Síndrome de abstinencia & 25 & --- & $8-42$ & --- & --- \\
\hline $\begin{array}{l}\text { Infecciones/Hemorragia digestiva/Pan- } \\
\text { creatitis }\end{array}$ & 16 & --- & $5-33$ & --- & --- \\
\hline Lesiones & 9 & --- & $2-25$ & --- & --- \\
\hline Otro & 6 & --- & $0-21$ & --- & --- \\
\hline Síntomas de ingreso & & & & 75 & $63-86$ \\
\hline $\begin{array}{l}\text { Temblores, sudoración, excitación, delirio } \\
\text { y/o insomnio }\end{array}$ & 27 & --- & $8-55$ & --- & --- \\
\hline $\begin{array}{l}\text { Diarrea, hemorragia digestiva o dolor } \\
\text { abdominal }\end{array}$ & 33 & --- & $12-62$ & --- & --- \\
\hline Varios & 13 & --- & $2-41$ & --- & --- \\
\hline Otras enfermedades & 89 & --- & $72-98$ & 53 & $40-67$ \\
\hline \multicolumn{6}{|l|}{ Entorno del paciente } \\
\hline Convivencia & & & & 37 & $24-50$ \\
\hline Solo & 21 & --- & --- & --- & --- \\
\hline Familia propia & 26 & --- & --- & --- & --- \\
\hline Familia origen & 32 & --- & --- & --- & --- \\
\hline Institucionalizado/Otro & 21 & --- & --- & --- & --- \\
\hline Continencia & 62 & --- & --- & 57 & $43-70$ \\
\hline Antecedentes familiares de consumo & & & & 63 & $50-76$ \\
\hline Un familiar directo & 36 & --- & $14-59$ & --- & --- \\
\hline Varios familiares & 50 & --- & $27-73$ & --- & --- \\
\hline Ninguno/Desconoce & 14 & --- & $3-35$ & --- & --- \\
\hline
\end{tabular}

Nota. ${ }^{\star}$ Los porcentajes fueron calculados con base en los registros con datos. IC = Intervalo de confianza. Elaboración propia. 
relacionadas. Se encontró una prevalencia mayor de datos referidos al consumo de alcohol que en otros estudios (Pechansky et al., 2005; Smothers et al., 2004), lo cual podría deberse a la existencia de una unidad especializada en alcoholismo en el hospital. No obstante, en muchos casos los registros fueron incompletos. Se evidencia así la necesidad de sistematizar la información señalizada en las historias clínicas. Una posible estrategia complementaria sería construir guías para los profesionales sanitarios, que incluyan información sobre el diagnóstico y el tratamiento de los trastornos por uso de alcohol, así como estimular su formación en el área mediante prácticas profesionales y cursos específicos. Además, preguntas esenciales sobre ambas cuestiones podrían ser incluidas en una historia clínica única (Ministerio de Salud de la Nación, s. f.).

Otro tema a tener en cuenta es la necesidad de clarificar cómo debe ser registrada cierta información. Por ejemplo, la edad de inicio de consumo promedio fue de casi 19 años, cuando otros estudios en Argentina indican que la edad de inicio de consumo más frecuente es entre los 12 y los 15 años (Cassola et al., 2005). En los registros observados, no fue posible determinar si se trataba de la edad de inicio de consumo - dato relevante para la predicción del desarrollo de un trastorno por uso de alcohol (DeWit et al., 2000) - o si se trataba de la edad en que los pacientes comenzaron a presentar problemas por uso de alcohol, cuyo riesgo incrementaría entre los 24 y los 36 años para quienes iniciaron su consumo en la adolescencia (Grant et al., 2001). Una información muy importante desde la perspectiva de un tratamiento y poco frecuente en los registros, es el consumo de otras sustancias psicoactivas. El consumir otras sustancias psicoactivas (incluido el tabaco) durante la adolescencia y juventud se ha visto asociado al desarrollo de dependencia del alcohol (Brook et al., 2002); y viceversa, el tener un trastorno por uso de alcohol se vio asociado a cumplir con al menos un criterio de trastorno por uso de otras sustancias psicoactivas, junto con otros trastornos mentales que tampoco fueron mencionados (Burns y Teesson, 2002). Esto remarca la necesidad del trabajo interdisciplinario en el propósito de discernir una posible comorbilidad y ofrecer tratamientos acordes con las necesidades de los pacientes.

Por otro lado, se halló que muchas de las condiciones en las que se encuentran los pacientes denotan dificultades para abordar un tratamiento. Mayormente desocupados y con un nivel de estudios inferior al secundario, muchos de ellos están solos y no cuentan con soporte familiar. Este dato no es menor, ya que se ha observado que en otras poblaciones contar con el apoyo de alguien cercano podría incentivar la búsqueda de ayuda (To y Date, 2005) y mantener una recuperación a largo plazo (Moos y Moos, 2007). Así mismo, en este estudio, muchos pacientes abandonaron el tratamiento y la principal causa fue retomar el consumo de alcohol.

Casi nueve de cada 10 pacientes registraron tener algún familiar con problemas el consumo de alcohol. Este hallazgo se vincula con la literatura disponible, ya que una historia familiar de trastornos por uso de alcohol se relacionaría con mayores criterios endosados de un trastorno por uso de alcohol (Lee et al., 2013).

La proporción de enfermedades concomitantes y de internaciones previas también es elevada. La mayoría de las internaciones previas tuvo que ver con el uso de alcohol o presentaba sintomatología que se ha visto asociada a la ingesta patológica de alcohol (World Health Organization, 2014). Así mismo, los síntomas de ingreso en las internaciones descritos en los registros se correspondieron con los síntomas del síndrome de abstinencia según el DSM-5 (American Psychiatric Association, 2013) y de consumo perjudicial según la Clasificación Internacional de Enfermedades-10 (World Health Organization, 1992). Se podría hipotetizar que estos pacientes concurrirían a la consulta en la unidad al ser detectados por el médico o cuando la presencia de otras patologías asociadas graves los motiva a buscar ayuda, lo cual refuerza la idea de la necesidad de fortalecer la detección temprana del consumo perjudicial.

Los grupos de autoayuda como A A parecen tener un rol importante en la rehabilitación. Fueron el recurso más utilizado antes y durante el tratamiento en la unidad. Es un recurso que suele ser recomendado por los mismos profesionales, según hemos conocido por comunicaciones personales dentro de la unidad. Esto puede explicarse por el funcionamiento propio de la unidad de alcoholismo, la cual mantiene como práctica el uso estratégico de este tipo de recursos.

Adicionalmente, quienes hicieron uso del servicio tuvieron en general una cobertura pública en salud, recibieron su tratamiento en el hospital público y la gran mayoría de ellos requería medicación. Se generan entonces gastos en el sistema de salud pública que podrían subsanarse empleando métodos de detección temprana validados en nuestro contexto, tales como el Test de Identificación de Trastornos por Uso de Sustancias (AUdit) (Cremonte, Ledesma, 
Cherpitel y Borges, 2010) e intervenciones breves, las cuales han demostrado ser efectivas en contextos sanitarios (Cremonte et al., 2013). De hecho, estudios en poblaciones hospitalarias del país han demostrado altas prevalencias de consumo de riesgo, mucho mayores a las encontradas en la población general (Cremonte, Cherpitel, Borges, Peltzer y Santángelo, 2010). Por ello, de cara a la implementación de políticas públicas, es menester apoyar iniciativas para detectar y tratar tempranamente los trastornos por uso de alcohol; así como diseñar y ejecutar investigaciones que esclarezcan muchas de las hipótesis aquí esbozadas.

\section{Conclusiones}

Desde el punto de vista de las políticas sanitarias, los resultados presentados indicaron un alto grado de registros incompletos o dispares, lo cual derivó en la necesidad de sistematizar la información que se recoge en las historias clínicas, ya sea en soporte físico o digital. A su vez, los resultados que evidencian la gran cantidad de patologías concomitantes, internaciones previas y el avanzado estado de los trastornos por uso de alcohol señalan la necesidad de implementar políticas públicas que permitan la detección e intervención temprana (i.e. intervenciones breves) en torno al consumo patológico de alcohol. Además de la conveniencia de estandarizar los procesos asistenciales para los trastornos por uso de alcohol mediante, por ejemplo, guías de prácticas clínicas que orienten una mejor y más pronta atención, y en las que se clarifique la necesidad de evaluar y registrar información sobre la edad de inicio de consumo, el consumo de otras sustancias psicoactivas o la presencia de indicadores de otros trastornos psíquicos. También podrían incluirse mayores contenidos sobre el diagnóstico y el tratamiento de los trastornos por uso de alcohol y otras sustancias en los currículos de formación profesional de las disciplinas vinculadas a la atención sanitaria. Esta realidad se hace extensible a otros países latinoamericanos, en donde el funcionamiento del sistema de salud sea similar, es decir, donde no existan registros unificados o con distintos estamentos de salud a niveles nacional, provincial y municipal.

Finalmente, cabe mencionar que el carácter descriptivo de este informe impide la generalización a otros servicios y otras poblaciones. No obstante, constituye un punto de partida para nuevas investigaciones en el ámbito hospitalario - más extensas y con un mayor número de casos-, y se espera que contribuya a mejorar las actuales prestaciones y a orientar futuras políticas sanitarias.

\section{Referencias}

American Psychiatric Association. (2013). The Diagnostic and Statistical Manual of Mental Disorders: DSM-5. Arlington County: APA.

Arzeno, M., Blanco, G., Caffarini, B., Cioppetini, G., Gates, H. y Latorre, M. (1994). Indicadores de gravedad en la población alcohólica atendida por el HIGA. Alcmeon, 3(2). Recuperado de http://www.alcmeon.com. ar/3/11/ a11_07.htm

Atkinson, R. M., Misra, S., Ryan, S. C. y Turner, J. A. (2003). Referral paths, patient profiles and treatment adherence of older alcoholic men. Journal of Substance Abuse Treatment, 25(1), 29-35. doi: 10.1016/S07405472(03)00048-5

Brook, D. W., Brook, J. S., Zhang, C., Cohen, P. y Whiteman, M. (2002). Drug use and the risk of major depressive disorder, alcohol dependence, and substance use disorders. Archives of General Psychiatry, 59(11), 10391044. doi: 10.1001/archpsyc.59.11.1039

Burns, L. y Teesson, M. (2002). Alcohol use disorders comorbid with anxiety, depression and drug use disorders: Findings from the Australian national survey of mental health and wellbeing. Drug and Alcohol Dependence, 68(3), 299-307. doi: 10.1016/S03768716(02)00220-X

Cassola, I., Pilatti, A., Alderete, A., y Godoy, J. C. (2005). Conductas de riesgo, expectativas hacia el alcohol y consumo de alcohol en adolescentes de la Ciudad de Córdoba. Revista Evaluar, 5, 38-54. Recuperado de http://revistas.unc.edu.ar/index.php/revaluar/article/ view/539/479

Cherpitel, C. J., Ye, Y., Bond, J., Rehm, J., Cremonte, M., Neves, O., ..., Giesbrecht, N. (2006). The effect of alcohol consumption on emergency department services use among injured patients: A cross-national emergency room study. Journal of Studies on Alcohol and Drugs, 67(6), 890-897. doi: http://dx.doi.org/10.15288/ jsa.2006.67.890

Child, C. G. (1964). The liver and portal hypertension. Filadelfia: Saunders.

Cremonte, M., Cherpitel, C. J. y Monteiro, M. (2013). Interventions to reduce alcohol-related injury in the emergency department: Screening, brief intervention and monitoring. En C. Cherpitel, G. Borges, N. Giesbrecht, 
M. Monteiro y T. Stockwell (Eds.), Prevention of alcohol-related injuries in the Americas: From evidence to policy action (pp. 158-168). Washington: Organización Panamericana de la Salud/Organización Mundial de la Salud.

Cremonte, M., Ledesma, R. D., Cherpitel, C. J. y Borges, G. (2010). Psychometric properties of alcohol screening tests in the emergency department in Argentina, Mexico and the United States. Addictive Behaviors, 35(9), 818-825. doi: 10.1016/j.addbeh.2010.03.021

Cremonte, M., Cherpitel, C. J., Borges, G., Peltzer, R. I. y Santángelo, P. R. (2010). Drinking patterns and DSMIV alcohol use disorders' criteria in Argentinean emergency department patients. Journal of Drug Addiction, Education, and Eradication, 6(3), 209-220.

DeWit, D. J., Adlaf, E. M., Offord, D. R. y Ogborne, A. C. (2000). Age at first alcohol use: A risk factor for the development of alcohol disorders. American Journal of Psychiatry, 157(5), 745-750. Recuperado de http:// www.ncbi.nlm.nih.gov/pmc/articles/PMC3442775/

Esper, L. H., Corradi-Webster, C. M., Carvalho, A. M. y Furtado, E. F. (2013). Mulheres em tratamento ambulatorial por abuso de álcool: caracteristicas sociodemograficas e clinicas. Revista Gaucha De Enfermagem, 34(2), 93-101.

Grant, B. F., Stinson, F. S. y Harford, T. C. (2001). Age at onset of alcohol use and DSM-IV alcohol abuse and dependence: A 12-year follow-up. Journal of Substance Abuse, 13(4), 493-504. doi :10.1016/S08993289(01)00096-7

Lee, S., Lee, B. C., Kim, J. W., Yi, J. S. y Choi, I. (2013). Association between alcoholism family history and alcohol screening scores among alcohol-dependent patients. Clinical Psychopharmacology and Neuroscience, 11(2), 89-95. doi: http://dx.doi.org/10.9758/cpn.2013.11.2.89

Marik, P. y Mohedin, B. (1996). Alcohol-related admissions to an inner city hospital intensive care unit. Alcohol and Alcoholism (Oxford, Oxfordshire), 31(4), 393-396.

Ministerio de Salud de la Nación. (s. f.). Historia Clínica para Adultos de Uso Ambulatorio. Recuperado de http:// www.msal.gov.ar/ent/index.php/ informacion-equipos-de-salud/herramientas-para-la-consulta

Monras, M., Ortega, L., Mondon, S., Balcells, M. y Gual, A. (2004). Enfermedades relacionadas con el consumo de alcohol: seguimiento a los dos años de la hospitalización. Medicina Clínica, 123(14), 521-526. doi: 10.1016/ S0025-7753(04)74584-4
Monras, M., Ortega, L., Mondon, S. y Gual, A. (2003). Alcoholismo en el hospital general: detección y tratamiento. Medicina Clínica, 121(19), 733-735. doi: 10.1016/S0025-7753(03)74078-0

Monteiro, M. G. (2007). Alcohol y salud pública en las Américas: un caso para la acción. Washington: Pan American Health Organization.

Moos, R. H. y Moos, B. S. (2007). Protective resources and long-term recovery from alcohol use disorders. Drug and Alcohol Dependence, 86(1), 46-54. doi: 10.1016/j. drugalcdep.2006.04.015

Nielsen, S. D. y Gluud, C. (1992). Physician's information about alcohol problems at hospitalisation of alcohol misusers. Alcohol and Alcoholism (Oxford, Oxfordshire), 27(6), 659-665. Recuperado de http://alcalc. oxfordjournals.org/content/27/6/659.long

Pechansky, F., Genro, V. K., Von Diemen, L., Kessler, F. H. P. y Da Silveira-Santos, R. A. (2005). References to alcohol consumption and alcoholism in medical records of a general hospital of Porto Alegre, Brazil. A comparison between samples with a 20-year gap. Substance Abuse, 25(2), 29-34. doi: 10.1300/J465v25n02_05

Proudfoot, H. y Teesson, M. (2002). Who seeks treatment for alcohol dependence? Findings from the Australian National Survey of Mental Health and Wellbeing. Social Psychiatry and Psychiatric Epidemiology, 37(10), 451-456.

Smothers, B. A., Yahr, H. T. y Ruhl, C. E. (2004). Detection of alcohol use disorders in general hospital admissions in the United States. Archives of Internal Medicine, 164(7), 749-756. doi: 10.1001/archinte.164.7.749

To, S. E. y Date, M. R. (2005). Alcoholism and pathways to recovery: New survey results on views and treatment options CME. Medscape General Medicine, 8(1), 1-7.

World Health Organization. (1992). The ICD-10 classification of mental and behavioural disorders: Clinical descriptions and diagnostic guidelines. Ginebra: Autor.

World Health Organization. (2014). Global status report on alcohol and health 2014. Ginebra: Autor. Recuperado de http://apps.who.int/iris/bitstream/10665/112736/1/ 9789240692763_eng.pdf

Zachry III, W. M., Grizzle, A. J. y Munch, E. A. (2002). Understanding the costs and treatment of alcohol abuse and dependence. (Practical Pharmacoeconomics). Formulary, 37(8), 407-416. 